\title{
Solving Routing Problem using Particle Swarm Optimization
}

\author{
Abhishek Toofani \\ M.Tech (Computer Science) \\ Dayalbagh Educational Institute \\ Dayalbagh Agra
}

\begin{abstract}
In a graph there are so many paths can exist from a source to a destination node. Among them finding optimal path is very difficult problem. It is an NP hard problem to find path in a graph. In this paper a swarm intelligence technique called Particle swarm Optimization is used to solve routing problem which give optimal path from graph. Here discrete mathematics is used to encode particle in PSO, which break search space in small search space and solve this discrete optimization.
\end{abstract}

\section{General Terms}

Artificial intelligence, Networking.

\section{Keywords}

Particle swarm Optimization, Swarm intelligence, Unicast routing.

\section{INTRODUCTION}

Finding a route from a graph is very hard. There are so many algorithms; techniques are available to solve this problem. In any graph there can be so many paths from a single point of source to a particular destination but it is very difficult to find any optimal path of minimum cost.

The word swarm intelligence [1] is come from artificial intelligence, where we analysis the behavior of ants, insects or birds etc. After analysis their behavior we try to use this behavior in computer science to solve different optimization problem. To use this social behavior of particle firstly we should convert it into some mathematical term, some equation, or some formula, and then we can use it in our problem.

There are various technique comes under swarm intelligence. In this paper PSO is used to solve. So many paper is published to solve routing problem via PSO and genetic algorithms [3] all use same technique but they are different in encoding of particle in discrete form. [4] Here a simple encoding technique is used to solve this problem which divide whole search space into small spaces and combine all solution to find optimal path

\section{PARTICLE SWARM OPTIMIZATION}

In PSO there are group of particles called swarm which move in search space to find shortest path from source to destination. Here every particle have a current position (position of particle existence in current time), personal best position (best position travel by each particle till now), global best position (best position of any particle among all particles in search space), velocity (personal velocity of each particle). In this technique every particle try to move towards the particle which is close to the solution and after some time all particle stop to particular solution which is optimal solution.

$v_{i} \leftarrow \omega v_{i}+\varphi_{p} r_{p}\left(p_{i}-x_{i}\right)+\varphi_{g} r_{g}\left(g-x_{i}\right)$

$x_{i} \leftarrow x_{i}+v_{i}$

Here $\mathrm{v}=$ velocity of particle

$\omega, \varphi_{p}, \varphi_{g}$ are control parameter which controls the behaviors and efficiency of particle. In this routing problem their values are 1 .

General Pseudo code of PSO:

randomly generate initial swarm

repeat

for each particle $i$ to max iteration

compute the best fitness value of function

update velocity

update best position

update global position

update position

end for

until termination criterion is met

\section{PSO TO SOLVE ROUTING}

To solve routing problem using PSO, first we have to make some change in general equations so that we can used it in our problem and we should do coding of particle so that it can solve discrete problem.

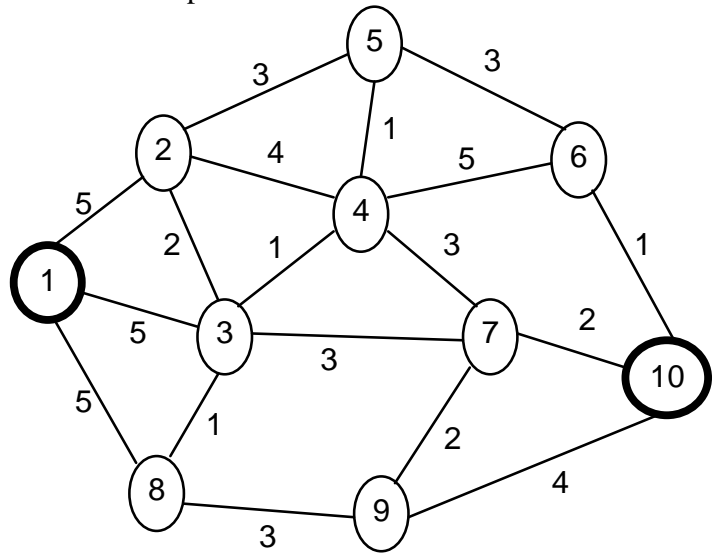

Fig 1: Demo network of 10 nodes where node 1 is source node and 10 is destination node.

Fig:1 show a demo network which is used to describe the logic of PSO to find route. In this Network Node 1 is source node and node 10 is destination node. As starting of PSO We 
have to initially define particles (means initially find random path from source to destination because every particle gives solution of problem). This logic gives more accurate result if particle are spread out over the network so far so that initially path can be found from all space of network. These initial paths break network in small search space. For example initially 3 particles are taken for this network (Network is small so number of particle is small but more number of particle give more accurate solution because more particles can cover more nodes and give more initial solution in all space). So initial particle value are...

$$
C_{\text {part }}[][]=\left[\begin{array}{cccccc}
1 & 2 & 3 & 4 & 6 & 10 \\
1 & 3 & 7 & 10 & & \\
1 & 8 & 9 & 7 & 10 &
\end{array}\right]
$$

Here $C_{\text {part }}$ a $2 \mathrm{D}$ array (or matrix can also be used) matrix which show initially define particle. Since as told earlier every particles have initial velocity so velocity of particle is define by $V_{\text {part }}$ (a $2 \mathrm{D}$ array vector), which is initially zero.

At the starting of algorithms initial paths are the current personal best paths of particles define by $P_{\text {best }}$ (a $2 \mathrm{D}$ array vector).

$$
P_{\text {best }}[][]=\left[\begin{array}{cccccc}
1 & 2 & 3 & 4 & 6 & 10 \\
1 & 3 & 7 & 10 & & \\
1 & 8 & 9 & 7 & 10 &
\end{array}\right]
$$

And the global best is the particles which have small cost among all particles define by $G_{\text {best }}$ (a single array vector).

$$
G_{\text {best }}[]=\left[\begin{array}{llll}
1 & 3 & 7 & 10
\end{array}\right]
$$

After initialization of particles updation is take place. Here it can be seen that difference of position gives us velocity and addition of position with velocity gives us new position. So in updation firstly velocity of particle if update by eq. 1.0 .

Since these particles are in discrete form so updation (addition, subtraction of velocity and position) cannot be take place as normal continuous value. So this updation is defined in discrete mathematics form for example third particle's velocity is updated as follows ...

$$
\begin{aligned}
V_{\text {part }}[3][] & =G_{\text {best }}[]-P_{\text {best }}[3][] \\
V_{\text {part }}[3][] & =\left\{\begin{array}{lll}
13 & 7 & 10
\end{array}-\left\{\begin{array}{lll}
1 & 89 & 10
\end{array}\right\}\right. \\
V_{\text {part }}[3][] & =\{3\}
\end{aligned}
$$

like this velocity of particles can be find as

$V_{\text {part }}[][]=\left[\begin{array}{l}6 \\ 0 \\ 3\end{array}\right]$

Every particle updates its position according to this new velocity. So updation of position of every particle is defined as...

$$
C_{\text {part }}[][]=V_{\text {part }}[][]+C_{\text {part }}[][]
$$

So for third particle we can find new position as...

$C_{\text {part }}[][]=\{3\}+\left\{\begin{array}{llll}189 & 9 & 10\end{array}\right\}$

$C_{\text {part }}[][]=\left\{\begin{array}{llllll}1 & 8 & 9 & 7 & 10 & 3\end{array}\right\}$

Fig: 2(a) shows the three $P_{\text {best }}$ via dark solid line and one $G_{\text {best }}$ via dark dashed line. After updation of second particle
Now this gives a set of nodes, now from this set of nodes we try to find a route from source to destination. One benefit of this approach is that we don't need to search all nodes of network we have to search path from these sets of node. So by doing this all network is broken into small network and we search within this small group of networks, these small networks are changed their node position dynamically whenever velocity and position of particles are changed and at the end all particle give one solution which is optimal solution.

$$
C_{\text {part }}[3][]=\left[\begin{array}{lllll}
1 & 8 & 3 & 7 & 10
\end{array}\right]
$$

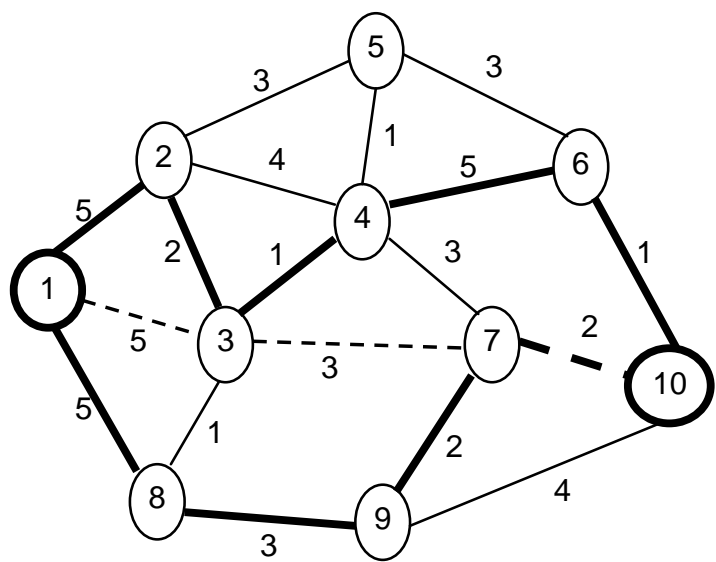

Fig 2(a): network shows $C_{\text {part }}, P_{\text {best }}, G_{\text {best }}$

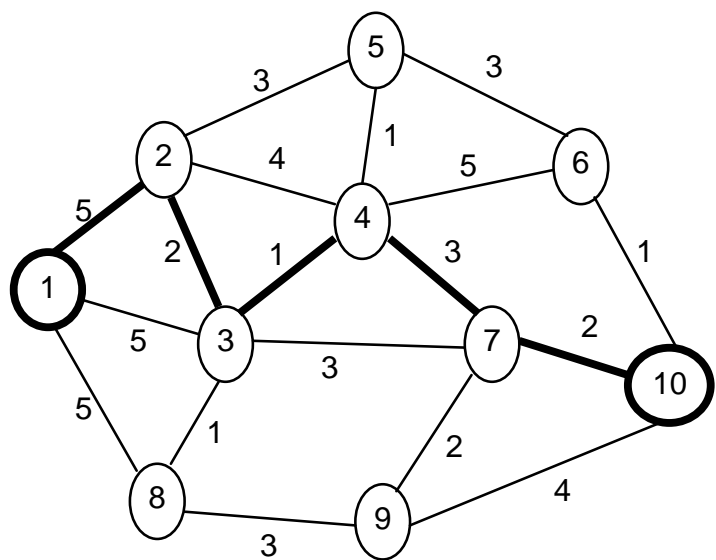

Fig 2(b): Network after updation of second particle.

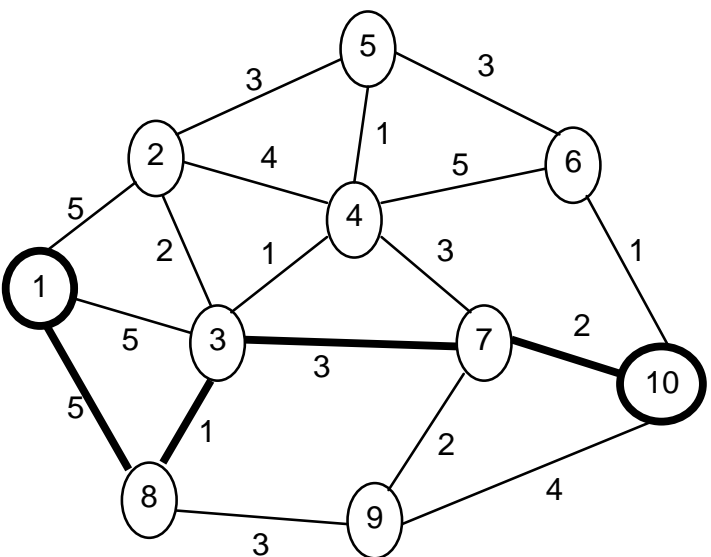

Fig 2(c): Network after updation of third particle

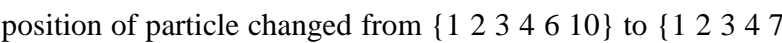
$10\}$, by doing this cost of path is reduced from 17 to 13 which 
is shown in fig: 2 (b).like this fig: 2(c) show path of third particle changed from $\left\{\begin{array}{llll}1 & 89 & 9 & 10\end{array}\right\}$ to $\left\{\begin{array}{l}183710 \\ 8\end{array}\right.$ and cost of path is reduced from 12 to 11 .

\subsection{Simulation result}

To check the working of logic, simulation is done. In this 35 nodes are taken, every link connected to the nodes has some cost.

Simulation is done in two ways to check the effect of particles. In first simulation 10 particles, 35 nodes and 50 iterations are used and in second simulation 15 particles, 35 nodes and 50 iterations are used.

Two figures (Fig: 3(a), 3(b)) are shown here as result of both simulation. Every graph is made between cost of link and number of iterations means it show total cost of paths on particular number of iteration.

In Fig: 3(a), it can be seen that at $5^{\text {th }}$ iteration the cost of path is 73 and after finish of 50 iteration the minim cost path is 27 which is optimal path of network. In second figure Fig: 3(b), cost of path is 56 on $5^{\text {th }}$ iteration because in second simulation number of particles are 15, so they spread of so far in network and find a good path in few iteration compare to previous iteration.

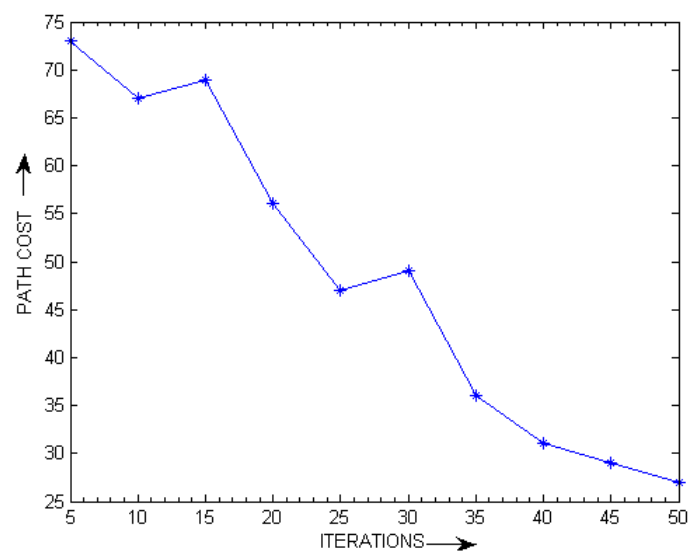

Fig 3(a): Simulation results when number of particles are 10.

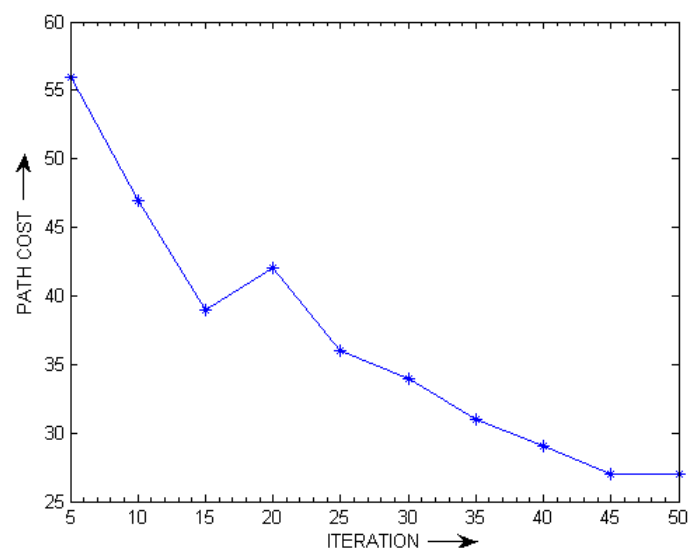

Fig 3(b): Simulation results when number of particles are 15.

\section{CONCLUSION}

Many Ideas are presented on solving routing problem using PSO, but this approach is different because it has simple encoding of particles and easy to implement. It gives batter result as particles or numbers of iteration are increased. It can also be used to solve multicast routing problem [6] or any type of routing problem because the main objective is to minimize the cost of path [11]. Not only routing other discrete problem can also be solved using this approach.

\section{REFERENCES}

[1] Kennedy, J. Bur. of Labor Stat., 1995, Proceedings. IEEE International Conference on neural network

[2] F. Xiang, L. Junzhou, W. Jieyi, G. Guanqun, 1999, QoS routing based on genetic.

[3] Chang Wook Ahn, Student, R. S. Ramakrishna, 2002, a genetic algorithm for shortesh path routing problem and sizing of populations, ieee transactions on evolutionary computation. Vol. 6 , no. 6 .

[4] Yiheng Xu1, Jinglu Hu, Kotaro Hirasawa, Xiaohong Pang, 2007, a new cooperative approach to discrete particle swarm optimization, SICE Annual Conference.

[5] Guoliang Xue,2003, minimum cost multicast and unicast routing in communication network, ieee transactions on communications, vol. 51 , no. 5 ,

[6] Rehab F. Abdel-Kader,2011, an improved discrete PSO with GA operators for efficient QoS- multicast routing, International Journal of Hybrid Information Technology Vol. 4, No. 2.

[7] E. Baburaj1, and V. Vasudevan2, 2008, An Intelligent Mesh Based Multicast Routing Algorithm for MANETs using particle Swarm Optimization, IJCSNS International Journal of Computer 214 Science and Network Security, VOL.8 No.5.

[8] N. Punnagai and P. Visu, 12 \& 13 April 2012, QoS Multicast routing for NP Completeness and PSO Optimization under Inaccurate Network Information, International Conference on Computing and Control Engineering (ICCCE 2012).

[9] Taoshen LI, Zhigang ZHAO, Zhihui GE, 2011, An Adaptive Swarm Optimization Algorithm for Anycast routing, Journal of Computational Information Systems 7:5 (2011) 1559-1566.

[10] Davoud Sedighizadeh and Ellips Masehian, 2009, Particle Swarm Optimization Methods,Taxonomy and application, International Journal of Computer Theory and Engineering Vol., No. 5.

[11] Pandhapon Sombuntham and Voratas Kachitvichayanukul, 2010, A Particl Swarm Optimization Algorithms for Multi-depot Vehicle Routing problem with Pickup and Delivery Requests. 